\title{
Study on the Guiding Mechanism of Whistler Radio Waves
}

\author{
Saburo Adachi \\ Department of Electrical Communications, Tohoku University, Sendai, Japan
}

\author{
(Received October 14, 1964; revised November 23, 1964)
}

\begin{abstract}
A full wave theory is applied to the whistler radio wave propagation along a plasma slab with an enhanced or depressed plasma density which is imbedded in an infinite magnetoplasma. Rigorous dispersion equation is solved for a thin slab in approximate but explicit forms. Three types of propagation modes are found: (a) a completely trapped surface wave mode along the depressed slab in the frequency region above a half of gyrofrequency and below a gyrofrequency, (b) a completely trapped surface wave mode along the highly enhanced slab in the frequency region above a half of gyrofrequency and below a certain cutoff frequency less than a gyrofrequency, and (c) a partially trapped (leaky) surface wave mode along the enhanced slab in the frequency region above a certain cutoff frequency and below a half of gyrofrequency. Dispersion properties, field distributions and an attenuation of the third mode due to the leakage of the transmitted power are discussed in detail. The attenuation is found to increase very rapidly with increasing frequency, thickness and enhancement of ionization of the slab. The exact numerical solutions are also obtained and compared with the approximate solutions.
\end{abstract}

\section{Introduction}

A number of theoretical investigations have been made on the propagation of whistler radio waves since the publication of Storey's famous paper [Storey, 1953], and not a few papers have dealt with the propagation path and the guiding mechanism of the whistlers. The present general idea accepted by many investigators on the guiding mechanism of the whistlers is that there may exist earth magnetic field-alined ducts in which the electron density is greater (or less) than the density outside, and the whistlers are guided along the ducts without loss due to the divergence of the wave. Concerning the guidance along such ducts, good review papers have been given by Storey [1962] and Booker [1963].

The ray theory has been used for the calculations of the whistler ray paths by Maeda and Kimura [1956], Smith et al. [1960] and Yabroff [1961]. Voge [1961, 1962] and Booker [1962] calculated the minimum discontinuity of ionization density and the corresponding minimum width of the ducts required for the trapping by using the analogous method with that used in the tropospheric duct propagation. These theories are considered to be appropriate for comparatively wide ducts. Northover [1959] has dealt with the guidance of the whistlers along an inhomogeneous cylindrical plasma column by use of the wave theory, in which he has expanded the fields into power series of the radial distance from the column axis.

Storey [1962] has raised a question whether very narrow columns or slabs can support trapped waves analogous to the surface wave modes along open transmission lines. In order to answer this question a full wave theory must be applied. In connection with this question, this paper deals with the electromagnetic wave propagation along a sharply bounded homogeneous infinite magnetoplasma slab which is imbedded in an infinite magnetoplasma of a different plasma density with that of the slab. A rigorous dispersion equation based on the classical magneto-ionic theory, Maxwell's equations and conventional boundary conditions is derived and solved first for a thin slab in approximate but explicit forms. These approximate solutions are partly compared with numerically obtained exact solutions. The existence of completely trapped and partially trapped waves, their dispersion properties, and an attenuation due to the leakage are discussed in detail, in connection with the guiding mechanism of the whistlers. 


\section{Model for Theoretical Analysis}

The earth magnetic field-alined plasma duct is represented by an infinite uniform plasma slab which is perpendicular to the $x$-axis and has a thickness of $2 a$ as shown in figure 1 . The magnetic field of the earth is assumed to be uniform in the whole region and parallel to the slab and $z$-axis. The corresponding electron gyrofrequency of the magnetic field is denoted by $\omega_{H}$. Let the electron plasma frequencies of the plasma slab and the surrounding plasma region be denoted by $\omega_{p}$ and $\omega_{p}^{\prime}$, respectively. In the present analysis the effect of ions is neglected for simplicity. The effect of finite compressibility of the plasma is also disregarded. This does not cause any error when whistler radio waves propagating parallel to the magnetic field are considered. The error when the propagating direction is not parallel to the field is not appreciable unless the electron temperature exceeds $10^{4}{ }^{\circ} \mathrm{K}$ [Kieburtz, 1964]. Thus the classical magneto-ionic theory is applied in this analysis.

\section{Electromagnetic Fields and Dispersion Equation}

The combined Maxwell's equation for an electric field $\vec{E}(x, y, z)$ is expressed by

$$
\left(\nabla \nabla-\nabla^{2} \hat{\hat{1}}-k_{0}^{2} \hat{\hat{\epsilon}}\right) \vec{E}=0,
$$

where the time dependence of $e^{j \omega t}$ is used and will be suppressed in the text, $k_{0}$ is a free space wave number, $\hat{\hat{1}}$ is the unit matrix, and $\hat{\hat{\epsilon}}$ is the well-known relative dielectric tensor of the plasma and is given by the form

$$
\hat{\hat{\epsilon}}=\left(\begin{array}{ccc}
\epsilon_{1} & -j \epsilon_{2} & 0 \\
j \epsilon_{2} & \epsilon_{1} & 0 \\
0 & 0 & \epsilon_{3}
\end{array}\right) .
$$

When the plasmas are assumed to be lossless, $\epsilon_{1}, \epsilon_{2}$, and $\epsilon_{3}$ (the plasma parameters inside the plasma slab) are expressed by

$$
\epsilon_{1}=1-\frac{\omega_{p}^{2}}{\omega^{2}-\omega_{H}^{2}}, \epsilon_{2}=\frac{\omega_{H}}{\omega} \frac{\omega_{p}^{2}}{\omega^{2}-\omega_{H}^{2}}, \epsilon_{3}=1-\frac{\omega_{p}^{2}}{\omega^{2}},
$$

where the sign of $\omega_{H}$ is determined as being identical to the sign of the d-c magnetic field. The plasma parameters $\epsilon_{1}^{\prime}, \epsilon_{2}^{\prime}$, and $\epsilon_{3}^{\prime}$ in the surrounding region are given by (3) only by replacing $\omega_{p}$ by $\omega_{p}^{\prime}$.

When the $z$-dependence of the electromagnetic fields is assumed to take the form of $e^{-j k o h z}$ ( $h$ is the relative axial propagation constant), the field components are obtained by a combination of two scalar functions which satisfy the wave equation [Kales, 1953]:

$$
\nabla_{t}^{2} \phi_{i}-s_{i}^{2} k_{0}^{2} \phi_{i}=0, \quad i=1,2
$$


where $s_{1}$ and $s_{2}$ correspond to relative transverse propagation constants (real part is an attenuation constant) of a left-handed plane wave and/or a right-handed plane wave (any combination depending on the value, $h^{2}$ ) for a Cartesian coordinate, and these two values are mutually coupled through the value $h^{2}: s_{1}^{2}$ and $s_{2}^{2}$ are the two solutions of the quadratic equation with respect to $s^{2}$ given by

$$
s^{4}-s^{2}\left[h^{2}\left(1+\frac{\epsilon_{3}}{\epsilon_{1}}\right)+\frac{\epsilon_{2}^{2}}{\epsilon_{1}}-\epsilon_{1}-\epsilon_{3}\right]+\frac{\epsilon_{3}}{\epsilon_{1}}\left[\left(h^{2}-\epsilon_{1}\right)^{2}-\epsilon_{2}^{2}\right]=0 .
$$

The relative propagation constants $s_{1}^{\prime}, s_{2}^{\prime}$ in the surrounding region also satisfy the equation of the same form with (5) in which $\epsilon_{1}, \epsilon_{2}$, and $\epsilon_{3}$ are replaced by $\epsilon_{1}^{\prime}, \epsilon_{2}^{\prime}$, and $\epsilon_{3}^{\prime}$. From this equation we obtain the following relations:

$$
\begin{aligned}
& s_{1}^{\prime 2}+s_{2}^{\prime 2}=h^{2}\left(1+\frac{\epsilon_{3}^{\prime}}{\epsilon_{1}^{\prime}}\right)+\frac{\epsilon_{2}^{\prime 2}}{\epsilon_{1}^{\prime}}-\epsilon_{1}^{\prime}-\epsilon_{3}^{\prime} \\
& s_{1}^{\prime 2} s_{2}^{\prime 2}=\frac{\epsilon_{3}^{\prime}}{\epsilon_{1}^{\prime}}\left[\left(h^{2}-\epsilon_{1}^{\prime}\right)^{2}-\epsilon_{2}^{\prime 2}\right],
\end{aligned}
$$

which relate the transverse propagation constants in the surrounding region to the axial propagation constant.

As is well known, the electromagnetic fields can neither be separated TE and TM modes nor $E$-symmetry modes and $H$-symmetry modes in general. For the present problem we obtain the electromagnetic fields in the following forms:

$$
\begin{aligned}
& E_{x}^{\mathrm{I}}=A\left(s_{1}^{\prime 2}+\Lambda^{\prime 2}+\frac{\epsilon_{2}^{\prime 2}}{\epsilon_{1}^{\prime}}\right) e^{-s_{1}^{\prime} k 0 x}+B\left(s_{2}^{\prime 2}+\Lambda^{\prime 2}+\frac{\epsilon_{2}^{\prime 2}}{\epsilon_{1}^{\prime}}\right) e^{-s_{2}^{\prime} k 0 x} \\
& E_{y}^{\mathrm{I}}=-j \epsilon_{2}^{\prime}\left(A e^{-s_{1}^{\prime} k 0 x}+B e^{-s_{2}^{\prime} k 0 x}\right) \\
& E_{z}^{\mathrm{I}}=j \frac{\epsilon_{1}^{\prime}}{\epsilon_{3}^{\prime} h}\left\{A s_{1}^{\prime}\left(s_{1}^{\prime 2}+\Lambda^{\prime 2}\right) e^{-s_{1}^{\prime} k 0 x}+B s_{2}^{\prime}\left(s_{2}^{\prime 2}+\Lambda^{\prime 2}\right) e^{-s_{2}^{\prime} k 0 x}\right\} \\
& H_{x}^{\mathrm{I}}=j \frac{\epsilon_{2}^{\prime} h}{Z_{0}}\left(A e^{-s_{1}^{\prime} k 0 x}+B e^{-s_{2}^{\prime} k 0 x}\right) \\
& H_{y}^{\mathrm{I}}=\frac{\epsilon_{1}^{\prime}}{Z_{0} h}\left\{A\left(s_{1}^{\prime 2}+\Lambda^{\prime 2}\right) e^{-s_{1}^{\prime} k 0 x}+B\left(s_{2}^{\prime 2}+\Lambda^{\prime 2}\right) e^{-s_{2}^{\prime} k 0 x}\right\} \\
& H_{z}^{\mathrm{I}}=-\frac{\epsilon_{2}^{\prime}}{Z_{0}}\left\{A s_{1}^{\prime} e^{-s_{1}^{\prime} k 0 x}+B s_{2}^{\prime} e^{-s_{2}^{\prime} k 0 x}\right\}, \\
& x \geqslant a \\
& \Lambda^{\prime 2}=\frac{\epsilon_{1}^{\prime 2}-\epsilon_{2}^{\prime 2}}{\epsilon_{1}^{\prime}}-h^{2}, \quad Z_{0}=\sqrt{\frac{\mu_{0}}{\epsilon_{0}}}
\end{aligned}
$$

for the upper half of the surrounding region (signs of $s_{1}^{\prime}$ and $s_{2}^{\prime}$ are changed for the lower half, $x \leqslant-a)$, and

$$
\begin{aligned}
& E_{x}^{\mathrm{II}}=C\left(s_{1}^{2}+\Lambda^{2}+\frac{\epsilon_{2}^{2}}{\epsilon_{1}}\right) \cosh s_{1} k_{0} x+D\left(s_{2}^{2}+\Lambda^{2}+\frac{\epsilon_{2}^{2}}{\epsilon_{1}}\right) \cosh s_{2} k_{0} x \\
& E_{y}^{\mathrm{II}}=-j \epsilon_{2}\left(C \cosh s_{1} k_{0} x+D \cosh s_{2} k_{0} x\right) \\
& E_{z}^{\mathrm{II}}=-j \frac{\epsilon_{1}}{\epsilon_{3} h}\left\{C s_{1}\left(s_{1}^{2}+\Lambda^{2}\right) \sinh s_{1} k_{0} x+D s_{2}\left(s_{2}^{2}+\Lambda^{2}\right) \sinh s_{2} k_{0} x\right\}
\end{aligned}
$$




$$
\begin{gathered}
H_{x}^{\mathrm{II}}=j \frac{\epsilon_{2} h}{Z_{0}}\left(C \cosh s_{1} k_{0} x+D \cosh s_{2} k_{0} x\right) \\
H_{y}^{\mathrm{II}}=\frac{\epsilon_{1}}{Z_{0} h}\left\{C\left(s^{2}+\Lambda^{2}\right) \cosh s_{1} k_{0} x+D\left(s_{2}^{2}+\Lambda^{2}\right) \cosh s_{2} k_{0} x\right\} \\
H_{z}^{\mathrm{II}}=\frac{\epsilon_{2}}{Z_{0}}\left(C s_{1} \sinh s_{1} k_{0} x+D s_{2} \sinh s_{2} k_{0} x\right) \\
|x| \leqslant a \\
\Lambda^{2}=\frac{\epsilon_{1}^{2}-\epsilon_{2}^{2}}{\epsilon_{1}}-h^{2},
\end{gathered}
$$

for the slab region, respectively. Equation (8) corresponds to the case where the transverse field components are symmetric with respect to the $y-z$ plane, while the axial field components are antisymmetric. This equation gives the modes which reduce to a whistler plane wave propagating in the $z$-direction when the thickness of the slab decreases to zero, or $\omega_{p}$ becomes equal to $\omega_{p}^{\prime}$. Another mode whose transverse field components are antisymmetric with respect to the $y-z$ plane are expressed by the same equation as (8) only by interchanging the hyperbolic sine functions and hyperbolic cosine functions.

By assuming the conventional boundary conditions to be valid at the interfaces, $x= \pm a$, we obtain the rigorous dispersion equation expressed as follows:

$$
\begin{array}{r}
2 \frac{\epsilon_{2} \epsilon_{2}^{\prime} \epsilon_{3} \epsilon_{3}^{\prime}}{\epsilon_{1} \epsilon_{1}^{\prime}} h^{2}\left(X_{1}-X_{2}\right)-\left(s_{1}^{\prime}+s_{2}^{\prime}\right)\left(s_{1}^{2}-s_{2}^{2}\right)\left(\epsilon_{3}^{\prime} X_{1} X_{2}+\epsilon_{3} s_{1}^{\prime} s_{2}^{\prime}\right)-\epsilon_{3}^{\prime}\left(s_{1}^{\prime} s_{2}^{\prime}-\Lambda^{\prime 2}\right)\left\{\left(s_{1}^{2}+\Lambda^{2}\right) X_{1}-\left(s_{2}^{2}+\Lambda^{2}\right) X_{2}\right\} \\
-\epsilon_{3}\left(s_{1}^{\prime 2}+s_{2}^{\prime 2}+s_{1}^{\prime} s_{2}^{\prime}+\Lambda^{\prime 2}\right)\left\{\left(s_{1}^{2}+\Lambda^{2}\right) X_{2}-\left(s_{2}^{2}+\Lambda^{2}\right) X_{1}\right\}=0,
\end{array}
$$

where

$$
X_{1,2}=s_{1,2} \tanh \operatorname{coth}\left(s_{1,2} k_{0} a\right)
$$

and where $X_{1,2}$ takes a hyperbolic tangent function for the case of symmetric transverse fields and a hyperbolic cotangent function for the case of antisymmetric transverse fields.

For later use the ratio of $B$ to $A$ is given below:

$$
\begin{aligned}
\Gamma=\frac{B}{A} \\
=-\frac{s_{1}^{\prime}\left(s_{1}^{2}-s_{2}^{2}\right)+\left(s_{1}^{2}+\Lambda^{2}\right) X_{2}-\left(s_{2}^{2}+\Lambda^{2}\right) X_{1}+\frac{\epsilon_{1}^{\prime} \epsilon_{2}}{\epsilon_{1} \epsilon_{2}^{\prime}}\left(s_{1}^{\prime 2}+\Lambda^{\prime 2}\right)\left(X_{1}-X_{2}\right)}{s_{2}^{\prime}\left(s_{1}^{2}-s_{2}^{2}\right)+\left(s_{1}^{2}+\Lambda^{2}\right) X_{2}-\left(s_{2}^{2}+\Lambda^{2}\right) X_{1}+\frac{\epsilon_{1}^{\prime} \epsilon_{2}}{\epsilon_{1} \epsilon_{2}^{\prime}}\left(s_{2}^{\prime 2}+\Lambda^{\prime 2}\right)\left(X_{1}-X_{2}\right)} e^{\left(s_{2}^{\prime}-s_{1}^{\prime}\right) k 0 a .} .
\end{aligned}
$$

It can be easily shown from (9) and (10) that, when the thickness of the slab decreases to zero, or $\omega_{p}$ approaches to $\omega_{p}^{\prime}, s_{1}^{\prime}$ becomes zero, and therefore $\Gamma$ and the axial field components vanish.

\section{Approximate Solutions for a Thin Slab}

In this section approximate but explicit solutions are derived for the slab whose thickness is small compared to a free space wavelength, and for the hyperbolic tangent mode $\left(X_{1,2}\right.$ in (9) takes the tanh function) which reduces to the whistler plane wave when $k_{0} a$ decreases to zero. 
If $k_{0} a$ is set equal to zero we have $s_{1}^{\prime}\left(\right.$ or $\left.s_{2}^{\prime}\right)=0$ from (9) for the tanh function, and therefore from (6) we obtain a whistler plane wave solution:

$$
\begin{aligned}
h_{0}^{2} & =\epsilon_{1}^{\prime} \pm \epsilon_{2}^{\prime} \\
s_{20}^{\prime 2} & = \pm \frac{\epsilon_{2}^{\prime}}{\epsilon_{1}^{\prime}}\left(\epsilon_{1}^{\prime} \pm \epsilon_{2}^{\prime}+\epsilon_{3}^{\prime}\right) .
\end{aligned}
$$

By making use of the above relation we can solve the dispersion equation approximately as a perturbation of a whistler plane wave propagating in the $z$-direction. Let $k_{0} a$ be sufficiently small so that the relation, $s_{1,2} k_{0} a \ll 1$ holds however large $s_{1,2}$ is. Neglecting the terms of $\left(k_{0} a\right)^{2}$ and the higher orders in (9), we obtain the following approximate dispersion equation:

$$
\epsilon_{3} s_{1}^{\prime} s_{2}^{\prime}\left(s_{1}^{\prime}+s_{2}^{\prime}\right)+k_{0} a f\left(s_{1}^{\prime}, s_{2}^{\prime}, s_{1}, s_{2}, h\right)=0
$$

where

$$
f=\epsilon_{3}^{\prime}\left(s_{1}^{\prime} s_{2}^{\prime}-\Lambda^{\prime 2}\right)\left(s_{1}^{2}+s_{2}^{2}+\Lambda^{2}\right)-\epsilon_{3} \Lambda^{2}\left(s_{1}^{\prime 2}+s_{2}^{\prime 2}+s_{1}^{\prime} s_{2}^{\prime}+\Lambda^{\prime 2}\right)-2 \frac{\epsilon_{2} \epsilon_{2}^{\prime} \epsilon_{3} \epsilon_{3}^{\prime}}{\epsilon_{1} \epsilon_{1}^{\prime}} h^{2} .
$$

Substitution of $s_{1}^{\prime}=0$ in (13) leads to a rather compact form:

$$
f_{0}=\mp \frac{\epsilon_{2}^{\prime} \epsilon_{3}^{\prime} \epsilon_{3}}{\epsilon_{1}^{\prime} \epsilon_{1}}\left\{\epsilon_{1}^{2}-\left(\epsilon_{1}^{\prime} \pm \epsilon_{2}^{\prime} \mp \epsilon_{2}\right)^{2}\right\}
$$

after lengthy algebraic calculations. Thus, $s_{1}^{\prime}$ is obtained from (12) and (14) as

$$
\begin{aligned}
s_{1}^{\prime} & =-\frac{f_{0}}{\epsilon_{3} s_{20}^{\prime 2}} k_{0} a \\
& =\frac{\epsilon_{3}^{\prime}}{\epsilon_{1}\left(\epsilon_{1}^{\prime} \pm \epsilon_{2}^{\prime}+\epsilon_{3}^{\prime}\right)}\left\{\epsilon_{1}^{2}-\left(\epsilon_{1}^{\prime} \pm \epsilon_{2}^{\prime} \mp \epsilon_{2}\right)^{2}\right\} k_{0} a .
\end{aligned}
$$

It should be noted here that (15) is not valid when $\left|s_{20}^{\prime 2}\right|$ is zero or too small, i.e., $\epsilon_{1}^{\prime} \pm \epsilon_{2}^{\prime}+\epsilon_{3}^{\prime} \simeq 0$ (actually this occurs at $\omega \simeq \omega_{H} / 2$ ). By making use of (15) and the relation that $s_{1}^{\prime}$ should satisfy (5) for the primed $\epsilon$ 's, we obtain $h^{2}$ :

$$
\begin{aligned}
h^{2} & =h_{0}^{2}+\frac{\epsilon_{1}^{\prime} \pm \epsilon_{2}^{\prime}+\epsilon_{3}^{\prime}}{2 \epsilon_{3}^{\prime}} s_{1}^{\prime 2} \\
& =\epsilon_{1}^{\prime} \pm \epsilon_{2}^{\prime}+\frac{\epsilon_{3}^{\prime}\left[\epsilon_{1}^{2}-\left(\epsilon_{1}^{\prime} \pm \epsilon_{2}^{\prime} \mp \epsilon_{2}\right)^{2}\right]^{2}}{2 \epsilon_{1}^{2}\left(\epsilon_{1}^{\prime} \pm \epsilon_{2}^{\prime}+\epsilon_{3}^{\prime}\right)}\left(k_{0} a\right)^{2},
\end{aligned}
$$

and therefore from (6) $s_{2}^{\prime 2}$ is obtained as

$$
s_{2}^{\prime 2}=s_{20}^{\prime 2}+\frac{1}{2 \epsilon_{1}^{\prime} \epsilon_{3}^{\prime}}\left\{\epsilon_{1}^{\prime 2}+\epsilon_{3}^{\prime 2} \pm \epsilon_{2}^{\prime}\left(\epsilon_{1}^{\prime}+\epsilon_{3}^{\prime}\right)\right\} s_{1}^{\prime 2} .
$$

In the preceding equations the upper sign corresponds to the perturbed wave mode of the left-handed plane wave and the lower sign corresponds to the one of the right-handed plane wave 
or the whistler mode when $\omega<\omega_{H}$. Now we shall restrict ourselves to the whistler type of mode and assume that

$$
\omega_{p}^{2}, \omega_{p}^{\prime 2} \gg \omega_{H}^{2}
$$

This assumption is considered to be valid in the magnetosphere at most cases. Under the condition of (18) $s_{1}^{\prime}$ and $s_{2}^{\prime 2}$ are expressed as follows:

$$
\begin{aligned}
& s_{1}^{\prime}=\frac{\left(X-X^{\prime}\right)\left\{X+X^{\prime}-Y\left(X-X^{\prime}\right)\right\}}{X(Y-2)} k_{0} a, Y \neq 2 \\
& s_{2}^{\prime 2}=\frac{Y X^{\prime}(2-Y)}{Y-1}+0\left(k_{0}^{2} a^{2}\right),
\end{aligned}
$$

where

$$
X=\frac{\omega_{p}^{2}}{\omega^{2}}, X^{\prime}=\frac{\omega_{p}^{\prime 2}}{\omega^{2}}, Y=\frac{\omega_{H}}{\omega} .
$$

By examining the behavior of the above two constants when the constitutive parameters $X$, $X^{\prime}$, and $Y$ are varied, we can know the existence of the trapped or partially trapped waves and their properties. Equation (19) shows that $s_{1}^{\prime}$ is a real number, at least in the first order approximation. In order that the waves are trapped along the slab, $s_{1}^{\prime}$ must be positive so that the fields decrease exponentially in the transverse direction outwards from the slab. From (19) we find that there exist three cases for $s_{1}^{\prime}>0$ :

$$
\begin{array}{ll}
\text { Case (i): } \quad X<X^{\prime} \text { and } \omega_{H / 2}<\omega<\omega_{H} \\
\text { Case (ii): } \quad X>3 X^{\prime} \text { and } \omega_{H / 2}<\omega<\omega_{c} \\
\text { Case (iii): } \quad X^{\prime}<X<3 X^{\prime} \text { and } \omega_{c}<\omega<\omega_{H / 2},
\end{array}
$$

where

$$
\omega_{c}=\omega_{H} \frac{X-X^{\prime}}{X+X^{\prime}}
$$

From (20) we find that

$$
s_{2}^{\prime 2} \begin{cases}>0, & \omega_{H / 2}<\omega<\omega_{H} \\ <0, & 0<\omega<\omega_{H / 2}\end{cases}
$$

It follows that $s_{2}^{\prime}$ is a real number, i.e., $s_{2}^{\prime}= \pm\left|s_{2}^{\prime}\right|$ for Cases (i) and (ii), and is an imaginary number, i.e., $s_{2}^{\prime}= \pm j\left|s_{2}^{\prime}\right|$ for Case (iii). It is possible to show from (5) that $s_{2}^{\prime}$ is a positive real number when $h^{2}$ is given by (16) for both Case (i) and Case (ii). Therefore we find that these two cases give the completely trapped surface wave modes whose fields decay exponentially outwards from the slab. In Case (i) the plasma density of the slab is less than that of the surrounding region. And Case (ii) corresponds to the case where the plasma density of the slab is greater than three times of that of the surrounding region. The upper cutoff frequency given by (22) of this mode increases with increasing enhancement of the plasma density of the slab.

In Case (iii) where the electron density of the slab is between one and three times of that of the surrounding region, an imaginary value for $s_{2}^{\prime}$ violates the principle of energy conservation since it represents a whistler plane wave outgoing from or incoming to the slab whereas $h^{2}$ is a positive real number representing a nonattenuating wave. In fact a pure imaginary value for $s_{2}^{\prime}$ with the other propagation constants retained as real numbers can not satisfy the rigorous dispersion equation (9). Thus (9) must be solved more rigorously as a complex dispersion equation 
in order to be consistent with the energy conservation. It is believed however that the negative values of $s_{2}^{\prime 2}$ represented by (17) or (20) are valid in the first order approximation for the phase constant, leading to the leaky surface wave which is partially trapped along the slab and leaks part of its energy continuously through propagation. The attenuation constant due to the leakage of the power and hence the imaginary part of $s_{2}^{\prime 2}$ is found to be in the order of $\left(k_{0} a\right)^{3}$ from the following discussion.

Without recourse to solving the exact dispersion equation for complex values of $h^{2}$, we may be able to obtain an approximate attenuation constant of the mode for Case (iii) by power consideration, that is, by obtaining the power transmitted along the slab and the power leaked from the slab. When $k_{0} a$ is sufficiently small compared with unity the transmitted power is substantially contributed by the field components associated with $s_{1}^{\prime}$ in the surrounding region. Thus we obtain the approximate transmitted power per unit length in the $y$-direction $(A=1)$ as

$$
P_{t} \simeq 2 \operatorname{Re} \int_{0}^{\infty}\left[E_{x} \times H_{y}^{*}-E_{y} \times H_{x}^{*}\right] d x \simeq 2 \frac{\epsilon_{2}^{\prime 2} h}{s_{1}^{\prime} k_{0} Z_{0}} .
$$

The transmitted power increases in inverse proportion to $s_{1}^{\prime}$ or $a$ for a constant $A$. The leaked power per unit axial length can be obtained by using the fields at a far distance from the slab where the field component associated with $s_{1}^{\prime}$ diminishes. Thus we obtain the approximate leaked power per unit area of the slab:

$$
P_{l}=2 \operatorname{Re}\left[E_{y} \times H_{z}^{*}-E_{z} \times H_{y}^{*}\right] x \rightarrow \infty \simeq-2 \frac{\epsilon_{2}^{\prime 2}}{Z_{0} h^{2}}\left(\epsilon_{1}^{\prime}-\epsilon_{2}^{\prime}+\epsilon_{3}^{\prime}\right)\left|s_{2}^{\prime}\right||\Gamma|^{2},
$$

in which equation the sign of $s_{2}^{\prime}\left(s_{2}^{\prime 2}<0\right)$ is determined as $s_{2}^{\prime}=-j\left|s_{2}^{\prime}\right|$ so that only outgoing (energy) wave from the slab is permitted from the physical reality: the phase is directed towards the slab conversely with the power in this case [Smith, 1960]. Thus, the normalized attenuation constant $\alpha$ with respect to $k_{0}$ is obtained as

$$
\alpha=\frac{P_{l}}{k_{0} P_{t}} \approx s_{1}^{\prime}|\Gamma|^{2}(Y-2) \sqrt{Y(Y-2)},
$$

where $\Gamma$ and $s_{1}^{\prime}$ are given by (10) and (19) respectively, and both are proportional to $k_{0} a(\ll 1)$, it follows therefore that $\alpha$ is proportional to $\left(k_{0} a\right)^{3}$. We find also by inspecting (26), (10) and (19) that $\alpha$ increases very rapidly when $Y$ decreases towards 2 .

\section{Exact Solutions}

The rigorous dispersion equation given by (9) has been numerically solved by using an electronic computer for Case (i): $\omega_{p}<\omega_{p}^{\prime}$ and $\omega_{H} / 2<\omega<\omega_{H}$, where the completely trapped surface wave modes have been found to exist in the preceding section. In the same frequency region the completely trapped nonattenuating surface waves whose transverse fields are antisymmetric with respect to $y-z$ plane (that is, coth function is taken as $X_{1,2}$ in (9)) are also found to exist, if the plasma frequency of the slab is below a certain critical value $\omega_{c}^{\prime}$ which is less than $\omega_{p}^{\prime}$ depending on the frequency. This mode shows the dispersion property quite similar to the tanh modes. The calculated values of $h^{2}$ are shown in figure 3 for the tanh function and in figure 4 for the coth function in the next section, where the approximate solutions obtained in the preceding section are compared with the exact solutions.

\section{Numerical Examples and Discussions}

Figure 2 shows the approximate phase constant for Case (i). The parameter is the thickness of the slab in terms of the plasma wavelength of the surrounding region. In this figure the numerically obtained exact values (only for a pure real $h$ ) are plotted by crosses and compared with the approximate values. The excellent agreement is found between them. Due to the vanishing 


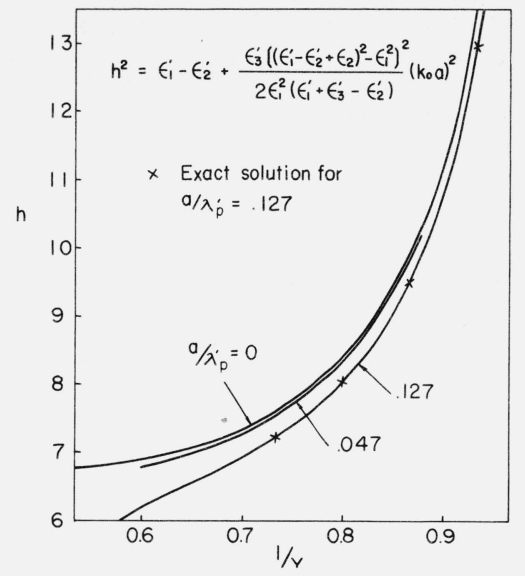

Figure 2. Approximate relative phase constant of the trapped wave mode (Case (i)), $\omega_{\mathrm{H}} / \omega_{\mathrm{p}}^{\prime}=0.3, \nu=\mathrm{X} / \mathrm{X}^{\prime}$ $=0.81, \lambda_{\mathrm{p}}^{\prime}$ is a free space wavelength corresponding to $\omega_{p}^{\prime}$.

of the denominator of the expression for $h^{2}$ at $\omega=\omega_{H} / 2$, the approximation is invalid near $1 / Y=0.5$, where the exact $h$ becomes complex.

In figure 3 the exact solutions obtained numerically for the same mode above (tanh mode) are plotted. We find from this figure that the moderate inhomogeneity due to the slab gives rise to considerable deviation of the phase constant from that of the plane wave. This is explained by the fact that most of the transmitted energy is confined in the slab with a plasma density less than that of the surrounding region.

Figure 4 shows the phase constant for the coth mode obtained by using a computor. The dispersion curves are quite similar to those of tanh mode in figure 3 , but show less deviation from the curve for the plane wave than in the case of tanh mode. This indicates less confinement of energy than the tanh mode as seen from the field configurations of these two modes.

Figure 5 shows the phase constant for Case (iii): the partially trapped (leaky) wave mode. The lower cutoff frequency $\omega_{c}$ given by (22) exists in this mode. Below this frequency $s_{1}^{\prime}$ is negative and the slab does not act as a guiding medium. But this does not necessarily imply that the propagation is forbidden: figure 7 shows that the attenuation decreases with decreasing frequency. The approximation is again invalid near the frequency, $\omega_{H} / 2$. We find that the deviation of the phase constant from the values for a plane wave is rather small compared to that in Case (i) (figure 2). This coincides with the fact that the wave is partially or loosely trapped to the slab.

Figure 6 illustrates the transverse field distributions of the partially trapped surface wave. The field amplitudes decreases almost exponentially and very gradually near the slab with ripples which are caused by the interference between the trapped wave component and the leaked wave component. The amplitude ratio of these two wave components is constant as determined by (10) to satisfy the boundary conditions. It follows therefore that the trapped wave component is continuously transformed into the leaky wave component through the mutual power between them, and this gives rise to an attenuation of the transmitted power. This mode is improper in the sense that the fields increase to infinity theoretically when $x \rightarrow \pm \infty$ if the surrounding plasma is lossless. This is a common nature of leaky waves. From figure 6 it is found that the waves are more strongly trapped with increasing frequency and thickness of the slab.

Finally figure 7 shows the attenuation of the partially trapped wave due to the leakage in nepers per one free space wavelength. We find that the attenuation increases with increasing frequency, thickness of the slab and enhancement of the plasma density of the slab. The thicker slab causes the stronger couping between the forementioned two wave components and results in greater attenuation. As an example, the attenuation is about $3.0 \mathrm{~d} B$ when $f=5 \mathrm{kc} / \mathrm{s}, f_{H}=50$ $\mathrm{kc} / \mathrm{s}, f_{p}^{\prime}=500 \mathrm{kc} / \mathrm{s}, \nu=X / X^{\prime}=1.03$, the slab of $0.34 \mathrm{~km}$ in width, and the propagation path of $10^{4} \mathrm{~km}$ are assumed. 


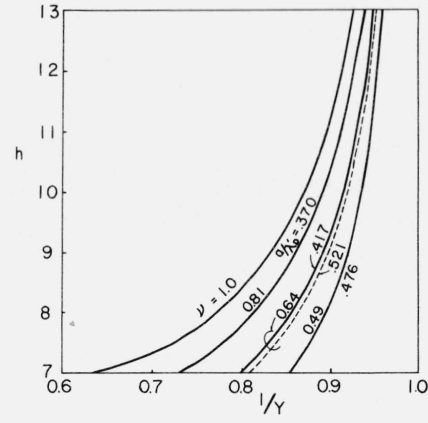

FIGURE 3. Exact relative phase constant of the trapped wave mode (tanh mode of Case $(i)), \omega_{\mathrm{H}} / \omega_{\mathrm{p}}^{\prime}=0.3$.

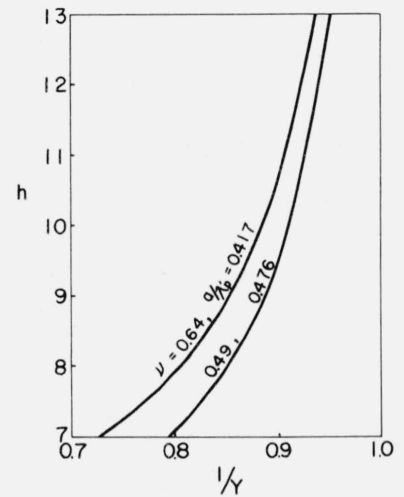

FIGURE 4. Exact relative phase constant of the trapped wave mode (coth mode of Case $(i)), \omega_{\mathrm{H}} / \omega_{\mathrm{p}}^{\prime}=0.3$.

FIGURE 7. Attenuation of the partially trapped wave mode due to the leakage of power in nepers per one free space wavelength, $\omega_{\mathrm{H}} / \omega_{\mathrm{p}}^{\prime}=0.1, \longrightarrow: \nu=1.03, \ldots \ldots .$. . $\nu=1.1$.

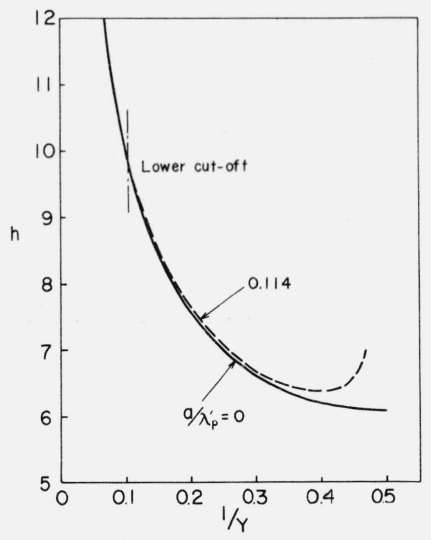

FIGURE 5. Approximate relative phase constant of the partially trapped wave mode (Case (iii)), $\omega_{H} / \omega^{\prime}$ $=0.33, \nu=1.23$.

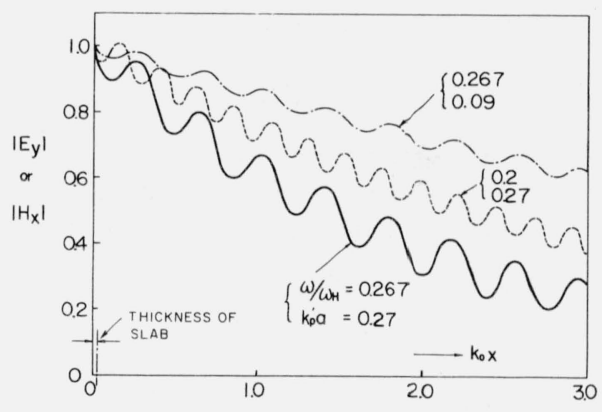

FIGURE 6. Field distributions of the partially trapped wave mode in the transverse direction, $\omega_{\mathrm{H}} / \omega_{\mathrm{p}}^{\prime}=0.33$, $\nu=1.23, \mathrm{k}_{\mathrm{p}}^{\prime}=2 \pi / \lambda_{\mathrm{p}}^{\prime}$.

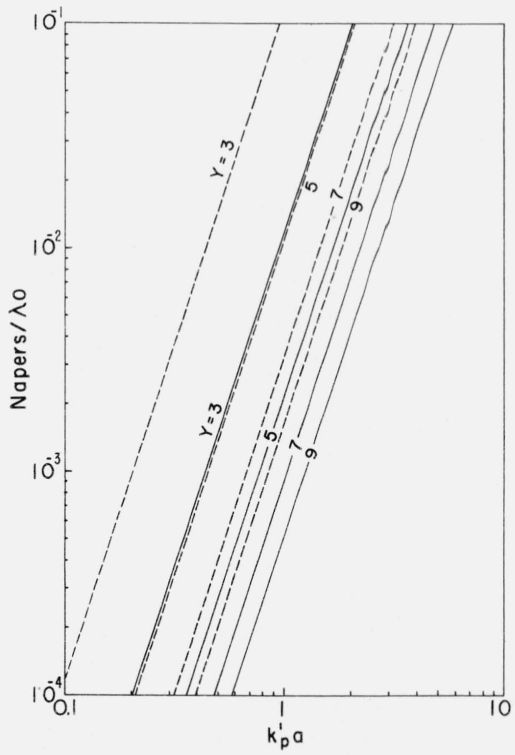




\section{Conclusion}

A full wave theory has been applied to the whistler radio wave propagation along a magnetoplasma slab with an enhanced or depressed plasma density which is imbedded in an infinite mag. netoplasma. The approximate but explicit solutions for the thin slab have been presented, and part of these approximate solutions have been confirmed by the numerically obtained exact solutions. The existence of the trapped or partially trapped waves and their dispersion properties, the fields, the attenuation due to the leakage and others have been discussed. The principal results obtained are as follows:

(i) The propagation of the completely trapped surface wave modes is possible for the slab of the depressed ionization in the frequency region of $\omega_{H} / 2<\omega<\omega_{H}$.

(ii) The same type of surface waves is propagated also along the slab whose plasma density is greater than three times of that of the surrounding plasma, in the frequency region of $\omega_{H} / 2$ $<\omega<\omega_{c}$.

(iii) In the frequency region of $\omega_{c}<\omega<\omega_{H} / 2$ only a partially trapped (leaky) surface wave mode is propagated along the slab of the enhanced ionization. The cutoff frequency $\omega_{c}$ depends on the degree of the enhancement of ionization.

(iv) The attenuation due to the leakage of the partially trapped wave increases very rapidly with increasing frequency, thickness, and enhancement of ionization of the slab.

The above results have been derived for the sharply bounded slab of homogeneously enhanced or depressed ionization because of relative simplicity of the analysis. It should be noted therefore that the quantitative conclusions in the above can not be strictly applied to an inhomogeneous slab with continuous boundaries, particularly when the thickness of the slab becomes appreciable in terms of a free space wavelength, and thus a different approach will be needed for such models [Wait, 1962].

The author expresses his gratitude to Y. Mushiake of the Tohoku University for his continuing interest and advice in the course of this work.

\section{References}

Booker, H. G. (Oct. 1962), Guidance of radio and hydromagnetic waves in the magnetosphere, J. Geophys. Res. 67, No. 11, 4135-4162.

Booker, H. G. (Sept. 1963), Guidance and beaming in the magnetosphere at hydromagnetic, audio and radio frequencies, General Assembly of URSI held in Tokyo, 1963.

Kales, M. L. (May 1953), Modes in wave guides containing ferrites, J. Appl. Phys. 24, No. 5, 604-608.

Kieburtz, R. B. (July 1964), VLF propagation in a compressible ionosphere, Radio Sci. J. Res. NBS/USNC-URSI 68D, No. 7, 795-805.

Maeda, K., and I. Kimura (Sept. 1956), A theoretical investigation on the propagation path of the whistling atmospherics, Rept. Ionospheric Research of Japan, X, No. 3, 105-123.

Northover, F. H. (Dec. 1959), The propagation of electromagnetic waves in ionized gases, Part I, II, III, IRE Trans. AP-7, Special Suppl. S340-S360.

Storey, L. R. O. (July 1953), An investigation of whistling atmospherics, Phil. Trans. Roy. Soc. A246, $113-141$.

Storey, L. R. O. (1962), Monograph on radio noise of terrestrial origin, p. 134-160, ed. by F. Horner (Elsevier Pub. Co., Amsterdam).

Smith, R. L., R. A. Helliwell, and I. W. Yabroff (Mar. 1960), A theory of trapping of whistlers in field-aligned columns of enhanced ionization, J. Geophys. Res. 65, No. 3, 815-823.

Smith, R. L. (Sept.-Oct. 1960), Guiding of whistlers in a homogeneous medium, J. Res. NBS 64D, (Radio Prop.), No. 5, 505-508.

Voge, J. (Nov.-Dec. 1961), Propagation guidée le long d'un feuillet atmosphérique ou (plus particulierement) exosphérique première partie, Ann. Télécomm., 16, 288.

Voge, J. (Jan.-Feb. 1962), Propagation guidée le long d'un feuillet atmosphérique ou (plus particulierement) exosphérique deuxième partie, Ann. Télécomm., 17, 34.

Yabroff, I. (Sept.-Oct. 1961), Computation of whistler ray paths, J. Res. NBS 65D, (Radio Prop.), No. 5, 485-505.

Wait, J. R. (1962), Electromagnetic waves in stratified media (Pergamon Press, Oxford). 